ÉGYPTE monde arabe

\section{Égypte/Monde arabe}

12-13 | 1993

Une économie en transition

\title{
Ajustement structurel et pauvreté. Introduction
}

\section{Marie-France Vernier}

\section{(2) OpenEdition}

\section{Journals}

Édition électronique

URL : https://journals.openedition.org/ema/1255

DOI : 10.4000/ema.1255

ISSN : 2090-7273

\section{Éditeur}

CEDEJ - Centre d'études et de documentation économiques juridiques et sociales

\section{Édition imprimée}

Date de publication : 31 mars 1993

Pagination : 101-108

ISSN : 1110-5097

\section{Référence électronique}

Marie-France Vernier, «Ajustement structurel et pauvreté. Introduction », Égypte/Monde arabe [En ligne], 12-13 | 1993, mis en ligne le 08 juillet 2008, consulté le 07 juillet 2022. URL : http:// journals.openedition.org/ema/1255; DOI : https://doi.org/10.4000/ema.1255

Ce document a été généré automatiquement le 7 juillet 2022.

Tous droits réservés 


\title{
Ajustement structurel et pauvreté. Introduction
}

\author{
Marie-France Vernier
}

1 Organisé sous les auspices du département d'économie de l'Université du Caire, un colloque sur le thème de «La réforme économique et son impact distributif a réuni dans la capitale égyptienne, du 21 au 23 novembre 1992, des économistes égyptiens et de diverses nationalités. Cette manifestation, qui intervenait plus d'un an après la mise en œuvre du programme de stabilisation et d'ajustement structurel de l'économie égyptienne ${ }^{1}$, a été l'occasion d'en dresser un premier bilan. Elle a notamment permis de montrer combien il est difficile de restructurer une économie dans le sens d'une plus grande liberté sans pour autant exclure du système de marché toute une partie de la population.

2 Deux thèmes étaient abordés : les effets probables de la réforme dans le domaine social - et notamment ses effets sur la pauvreté - et l'expérience d'économies ayant déjà appliqué des programmes analogues. Les débats suscités par les communications ont essentiellement porté sur les outils de la réforme et son impact sur la distribution des revenus.

3 Après avoir brièvement passé en revue ces communications, dont certaines sont présentées dans ces pages ${ }^{2}$, nous évoquerons les débats dominants et nous nous interrogerons sur la place accordée à l'État au sein du programme de stabilisation et d'ajustement structurel.

$4 \mathrm{Au}$ cours de la première journée, consacrée aux aspects sociaux de la réforme économique, H. Coulombe, H. A. Mac Kay et G. Pyatt ont analysé, dans une communication intitulée "Mesure des changements du bien-être des ménages : théorie et application en Côte-d'Ivoire (1985-1988) ", la capacité de différents indicateurs à rendre compte des variations du bien-être. Retenant un indicateur qui rapporte les dépenses aux besoins des ménages, ils ont conclu, grâce à l'application de courbes de Lorenz $z^{3}$ pour la période considérée, au déclin du bien-être de ceux-ci.

5 Outre les études réalisées par Heba Nassar («Quelques conséquences sociales du programme d'ajustement structurel en Égypte») et Mona al-Baradi'i («Impact du 
programme de redressement économique sur une répartition équitable des chances de scolarisation en Égypte »), publiées dans ces pages et dont nous précisons plus loin la teneur, A. 'Abd al-Latif, sous le titre «Une estimation du Fonds social de développement égyptien à la lumière de l'expérience bolivienne ", a mis en évidence les éléments susceptibles de faire obstacle au projet égyptien, au nombre desquels, selon elle, la composition pléthorique du conseil d'administration du Fonds et son indépendance décisionnelle limitée vis-à-vis de ses donateurs et des bénéficiaires des projets.

6 Le cas de quelques pays (Turquie, Ghana, Chili, Mexique, Tunisie et Allemagne réunifiée) ayant déjà appliqué une réforme a été abordé lors de la seconde journée. Sous l'intitulé « La réforme économique orthodoxe et son impact distributif : le cas de la Turquie », K. Borotav a dressé un bilan pessimiste d'une réforme réalisée dix ans plus tôt : diminution du bien-être des ménages, persistance des comportements rentiers, essor du clientélisme, croissance des activités non productives par rapport aux autres activités, détérioration des termes de l'échange agricole et stagnation de la productivité. Il en a conclu à la diminution du potentiel de croissance de l'économie turque.

7 L'étude du G. Boadi, intitulée "Neuf ans d'ajustement économique au Ghana: une première évaluation des impacts ", n'est pas moins sombre. Malgré la reprise de la croissance, l'amélioration des infrastructures sociales et économiques et la croissance de l'offre, le programme de stabilisation et d'ajustement structurel mis en place en 1983 n'a pas vraiment réussi, contrairement aux appréciations des instances internationales. L'inflation persiste, la dette étrangère jusqu'alors réduite s'est élevée, le coût de la vie et le chômage ont augmenté.

8 L'expérience chilienne, abordée par S. Maatouk sous le titre «Réforme économique au Chili et son impact distributif » (période 1973-1982), conduit, quant à elle, à un constat nuancé, faisant notamment état d'une baisse de l'inflation contrebalancée par une aggravation des inégalités et du chômage, tandis que la spéculation, ajoutée au découragement des investisseurs, freine le secteur productif.

9 Le bilan de la réforme au Mexique tel que le présente A. al-Mahdy dans son étude intitulée «Politiques de redressement économique au Mexique et ses impacts distributifs ", est également nuancé : si l'on enregistre, d'un côté, une croissance du PIB et une baisse de l'inflation et des transferts vers l'extérieur, d'un autre côté, les distorsions de la distribution se sont accentuées. Entre 1982 et 1990, les salaires réels, les revenus par tête et le PIB par tête ont diminué.

10 Pour Bou Ghazal en revanche («L'expérience de la réforme économique tunisienne ») la situation tunisienne s'est améliorée. Engagée dans un programme de stabilisation et d'ajustement structurel en 1986 après avoir tenté de mettre en place, seule, une politique de redressement économique, la Tunisie jouit d'une légère reprise économique confortée par de meilleurs résultats à l'exportation. Malgré un taux de chômage plus élevé et une baisse des salaires réels, les dépenses par tête évoluent favorablement et on observe une certaine diminution de la pauvreté.

11 L'expérience allemande est particulière. Présentée par A. 'Abd al-Wahab, la « Réforme économique dans les nouveaux États de la République Fédérale d'Allemagne » vise à transformer une économie planifiée en une économie régie par les lois du marché. Le pouvoir monétaire est ainsi transféré à la Banque centrale, le contrôle des changes est supprimé et les entreprises publiques privatisées. Mais les privatisations s'avèrent 
extrêmement coûteuses, le produit de la vente d'une entreprise ne compensant pas les coûts liés aux restructurations et aux indemnités. Deux ans après la réunification, l'exAllemagne de l'Est est confrontée au chômage, à la hausse du coût de la vie et à une chute du PIB.

C'est à la répartition des revenus de la population égyptienne et au développement financier qu'a été consacrée la dernière journée. Sous le titre "Questions méthodologiques sur l'utilisation des données des ménages pour l'évaluation de l'ajustement structurel», I. Sirag al-Din et S. Wahba ont tenté une estimation de l'incidence du programme de stabilisation sur la pauvreté. Utilisant pour cela un indicateur de pauvreté qui met en évidence les différences géographiques et les caractéristiques socio-économiques des ménages de Côte-d'Ivoire, puis le combinant avec un modèle d'équilibre général, ils ont montré la faiblesse des programmes dits de transferts ${ }^{4}$ ciblés sur la pauvreté. En effet, même si ces programmes, dans un premier temps, diminuent la pauvreté, ils ne semblent pas, à long terme, la réduire de manière significative en raison de la sensibilité de l'indicateur aux effets de fuite. Ainsi, d'une part, les pauvres bénéficient de transferts mais paient des impôts directs et indirects et d'autre part, y compris dans le cas d'une politique de transferts ciblée sur un groupe d'individus, la pauvreté subsiste.

13 Dans une seconde étude (publiée ci-après), intitulée «Evaluation de la pauvreté en Égypte en fonction des données des ménages ", $\mathrm{H}$ al-Laithy et $\mathrm{H}$. Kheir al-Din ont mis en évidence, à travers une analyse spatio-temporelle, les inégalités régionales et l'aggravation récente de la pauvreté. Nous y reviendrons à la fin de cette introduction.

Selon M. Muhyi al-Din, auteur d'une contribution intitulée « Le secteur financier entre la répression et la libéralisation ", il n'existe aucune confirmation théorique ni pratique de l'impact positif de la libéralisation financière sur l'épargne par le biais du taux d'intérêt. Dans de nombreux pays du Tiers Monde, et en Amérique Latine en particulier, l'épargne n'a pas été davantage mobilisée: on a surtout assisté à une redistribution de cette épargne entre les différents canaux existants. Quant à l'impact de la libéralisation sur l'investissement, il a été essentiellement négatif.

Trois thèmes ont dominé le débat qui suivait la présentation des communications : les conséquences attendues de la réforme sur la distribution des revenus, la faiblesse de l'épargne et les taux d'intérêt. La majorité des intervenants a estimé que la réforme aurait un impact défavorable pour les plus démunis. Citons les opinions les plus révélatrices.

La réforme, affirme S. al-Naggar, vise à court terme à lutter contre l'inflation, fût-ce aux dépens de la croissance. La question de la distribution est secondaire par rapport à cet objectif. Critiquant au contraire le programme de stabilisation et d'ajustement structurel, Muhammad Dwidar considère que la répartition des revenus est un élément essentiel de l'activité économique elle-même. En effet, selon les keynésiens, la répartition des revenus n'est pas neutre : elle influence le niveau de production et le niveau de consommation. Aussi n'est-il pas certain que la réforme permette le retour de la croissance.

17 Par ailleurs, Galal Amin, réagissant à la communication sur le Fonds social bolivien, met en avant l'insuffisance des moyens financiers du Fonds social égyptien pour contrecarrer les effets négatifs escomptés sur la population. Selon lui, la finalité de ce Fonds est moins économique que politique, l'objectif réel étant d'alléger les coûts 
politiques des licenciements que ne manqueront pas de provoquer les privatisations de quelques grandes industries.

Le débat s'est également porté sur les moyens de combattre la faiblesse de l'épargne ${ }^{5}$. Outre l'argument, soutenu par les auteurs de la réforme, selon lequel l'élévation des taux d'intérêt favorise l'augmentation de l'épargne, deux propositions ont été avancées. Selon $\mathrm{H}$. al-Sayeh, les privatisations devraient permettre une augmentation de l'épargne dont bénéficierait le secteur privét. Selon G. 'Abd al-Khaliq, la révision du système d'imposition devrait s'inscrire dans le cadre de cette réforme pour permettre une hausse de l'épargne, que menace la libéralisation fiscale. L'élévation des taux d'intérêt, conséquence attendue de la libéralisation, n'est donc pas suffisante.

Ces taux ont un rôle stratégique au sein de la nouvelle politique monétaire. Librement déterminés par le marché, ils ont considérablement augmenté ${ }^{7}$. Les conséquences escomptées par les auteurs de la réforme sont notamment une meilleure répartition des investissements, l'élévation du taux de l'épargne et une diminution de la fuite des capitaux.

H. al-Sayeh considère cependant que l'élévation des taux d'intérêt décourage l'État de recourir à l'emprunt, ce qui peut entraîner une diminution de la dette intérieure, renforcer la monnaie nationale vis-à-vis des devises étrangères et décourager la fuite des capitaux. En revanche, G. 'Abd al-Khaliq s'inquiète de cette libéralisation dans laquelle il n'est pas tenu compte de la structure du système bancaire, et s'interroge sur la capacité de celui-ci à gérer la nouvelle situation.

21 Le bilan du colloque est globalement pessimiste quant aux conséquences probables de la réforme sur la distribution des revenus. Les expériences présentées par les intervenants conduisent au constat nuancé d'une amélioration souvent partielle de l'économie mais d'une dégradation sociale. Ce constat est d'autant plus alarmant que la situation initiale n'est pas brillante en Égypte, comme en témoignent les articles sélectionnés ici et traitant de l'éducation, des effets sociaux et de la pauvreté. Néanmoins, la libéralisation ne semble pas remise en cause par les intervenants. Curieusement, alors que cette réforme prévoit un retrait progressif de l'État du secteur économique, les interventions ont peu porté sur cette question. La réforme s'attache à étendre les lois du marché au sein de l'économie; c'est ainsi, selon ses auteurs, que celle-ci pourra retrouver les bases d'une croissance autonome. Or, la suppression des subventions, les privatisations et la libéralisation du commerce extérieur auront des conséquences sur les conditions de vie des plus pauvres. De par son rôle dans la redistribution des revenus, l'État est le premier concerné. Aussi nous semble-t-il nécessaire de faire quelques remarques sur l'intervention de l'État dans un pays en voie de développement tel que l'Égypte.

22 L'engagement actuel de l'État est une survivance de la politique économique de l'époque nassérienne. Après la révolution de 1952, l'État a décidé d'assurer à tous l'accès à l'éducation et aux services de santé et de garantir une répartition équitable des revenus: nationalisations, réforme agraire, subventions, embauche systématique des diplômés de l'université par l'État..., sont les aspects principaux de cette politique. L'implication de l'État égyptien dans l'économie est donc profonde. Elle explique le type d'insertion de l'économie égyptienne dans les échanges mondiaux. Jusqu'en 1974, les relations commerciales, financières et politiques de l'Égypte concernaient principalement les pays de l'Est. Avec l'ouverture économique, des échanges s'établirent vers l'Occident. Cette implication s'inscrit dans le cadre du processus de 
formation d'une économie de marché, l'État étant «le lieu de diffusion des rapports marchands et capitalistes nécessaires à la réalisation de la division internationale du travail $»^{8}$.

En réalité, la libéralisation économique actuellement en cours est un processus ancien". Elle nous amène à nous interroger sur les relations qu'entretiennent les aspects sociaux et les aspects économiques d'une société. Etroitement liés, ils ne peuvent être considérés séparément. Or l'expérience de la réforme économique semble se solder dans un premier temps par l'apparition - ou l'aggravation - d'un dualisme au sein de la société, seule une partie de la population pouvant profiter des effets de l'amélioration de l'économie.

Aussi le rôle de l'État paraît-il essentiel pour permettre aux exigences du social et de l'économique de s'accorder. Il s'agit moins de pallier les insuffisances du marché vis-àvis de la population que de concevoir une dynamique «harmonieuse » entre ces deux domaines. Nous entendons ainsi une dynamique dans laquelle une partie de la population n'est pas marginalisée. Ainsi, quand l'économie de marché s'est installée dans les pays aujourd'hui industrialisés, la protection sociale s'est étendue, ces deux mouvements contemporains se réalisant dans des sociétés où l'État s'implique dans le domaine de l'économie et dans le domaine social ${ }^{10}$. Actuellement, dans les économies de marché qui constituent la référence pour l'élaboration d'une réforme, l'État est un acteur essentiel de l'activité économique.

Aussi, s'il semble effectivement nécessaire de modifier la dynamique sur laquelle repose l'économie, il est nécessaire d'en considérer en même temps les aspects sociaux et économiques. Jusqu'à présent, l'intervention de l'État s'est soldée par un déficit budgétaire persistant qui anticipe un prélèvement sur les ressources, une extension des comportements rentiers de type clientéliste et des entreprises publiques en sur-effectif. Son engagement ancien vis-à-vis des populations défavorisées n'a pas empêché une aggravation de la pauvreté ces dernières années. Certes, l'Égypte n'a pas le monopole de la contradiction entre une volonté affichée d'assurer une équité sociale et sa concrétisation. Seulement, il n'est pas certain que le retrait de l'État résolve la crise que traverse l'Égypte, d'autant que la diminution des dépenses publiques signifie que l'État réduit ses moyens d'intervention auprès de la population. L'affaiblissement de cette intervention s'est traduit, ces dernières années, par l'apparition d'associations dans les domaines de la santé et de l'éducation. L'expérience de l'Amérique Latine pourrait être utile. Les programmes de stabilisation et d'ajustement structurel se sont soldés par une persistance de la crise et un État poreux.

« Le retrait de l'État de certaines dépenses conduit à une absence d'État. Là où il est moins présent se mettent en place d'autres formes de pouvoir remplissant en partie les fonctions laissées en friche par l'État et/ou celles correspondant aux nouvelles demandes d'État... En partie rendus légitimes par leur réalisation, ils s'imposent. ${ }^{11}$

L'État cède ainsi quelques-unes de ses prérogatives et perd alors des éléments de sa légitimité.

Le programme de stabilisation et d'ajustement structurel, dont l'objectif est strictement économique, est mis en œuvre dans une société confrontée à une situation sociale difficile. Les articles publiés dans ce numéro sont révélateurs de l'état de la réflexion sur ce thème chez les économistes égyptiens. prix, la santé et l'éducation, l'étude de H. Nassar analyse avec précision les effets 
sociaux escomptés selon la place de chaque individu dans la société. Quant à l'étude de M. al- Baradi'i, elle dresse tout d'abord le constat de l'inégalité des individus devant l'accès à l'enseignement et, anticipant les effets aggravants du programme d'ajustement structurel, elle relève la contradiction d'une réforme dont la finalité affichée est de «mettre en valeur l'élément travail».

La société égyptienne est également confrontée à une pauvreté croissante, peut-être le défi essentiel qu'elle ait à affronter. Deux articles permettent d'évaluer le coût d'une politique ciblée. L'étude de $\mathrm{H}$. al-Laithy et $\mathrm{H}$. Kheir al-Din - effectuée sur la base des enquêtes "Budget des ménages" de 1974/75 et 1981/82 et sur les résultats préliminaires de l'enquête sur les revenus et dépenses de 1990/91, réalisées par le CAPMAS - propose une comparaison régionale et temporelle ainsi qu'une caractérisation du comportement des individus définis comme pauvres, à partir d'un " seuil de pauvreté relative " exprimé en dépenses moyennes par tête, seuil au-dessous duquel un individu est qualifié de pauvre. Les auteurs, dans une approche microéconomique, évaluent alors le coût et l'efficacité d'une politique ciblée.

Nous avons jugé intéressant d'ajouter à cet ensemble la contribution de N. Fergany qui, bien que n'ayant pas participé au colloque, a réalisé une étude dont le thème est proche de ceux évoqués plus haut. Dans le but d'évaluer le coût d'intervention du Fonds social de développement, N. Fergany détermine à un niveau très fin la population considérée comme vulnérable selon un ensemble de critères lui permettant d'identifier la pauvreté. Tout en observant la même réalité que les deux auteurs précédemment cités, il utilise des outils différents et se place dans une perspective macro-économique. Il aboutit de ce fait à des conclusions quelque peu différentes. Une comparaison régionale montre une répartition très inégale de la pauvreté, plus forte en zones rurales, notamment en Haute-Égypte.

31 La croissance de l'inégalité sociale dans un pays où l'État est amené à réduire son intervention constitue un défi pour les tenants de la réforme. Celle-ci saura-t-elle empêcher l'aggravation du dualisme au sein de la société égyptienne?

\section{NOTES}

1. Le programme de stabilisation et d'ajustement structurel a été mis en place en coopération avec le Fonds monétaire international (FMI) à partir de mai 1991. Cependant, dès mars 1990, le gouvernement égyptien a commencé la mise en oeuvre de réforme allant dans le sens des mesures préconisées par le FMI. Voir BLIN L., «Le programme de stabilisation et d'ajustement structurel de l'économie égyptienne ", Égypte/Monde arabe n 9, p. 16.

2. Pour des raisons techniques et des contraintes de place, nous n'avons pas reproduit dans ces articles l'intégralité des tableaux et graphiques qui figuraient dans les versions originales des communications, et nous avons parfois réduit certains développements. Que les auteurs de ces contributions ne nous en tiennent pas rigueur. Les traductions (de l'arabe et de l'anglais) ont été effectuées par Michel Galloux, Néfissa al-Bakly et Mona Badawi.

3. Les courbes de Lorenz sont ici utilisées pour mettre en évidence l'évolution de la concentration de l'indicateur sur une ou plusieurs périodes. 
4. Ces programmes consistent en transferts directs en faveur de la population ou subventions sur les prix.

5. Pour l'année fiscale 1989-1990, l'épargne représentait $11 \%$ du PIB. Voir BLIN L., op. cit. p. 42

6. Cet argument se fonde sur l'attente d'une meilleure performance financière des entreprises nouvellement privatisées.

7. Le taux d'adjudication des bons du Trésor, taux directeur du système bancaire, était de $19,5 \%$ au troisième trimestre 1991. Voir BLIN L. op. cit. p. 19.

8. SALAMA P. et MATHIAS G., L'État surdéveloppé. Des métropoles au Tiers Monde, La Découverte, Maspero, Paris, 1983, p. 39.

9. Pour plus de détails, voir CLEMENT F., Introduction au dossier « Vers une économie libérale ?» Égypte/Monde arabe $\mathrm{n}^{\circ}$ 9, p. 8.

10. POLANYI K., La grande transformation, Gallimard, Paris, 1944, p. $180 \mathrm{s.}$

11. SALAMA P. et VALIER J., L'économie gangrenée, La Découverte, 1990.

INDEX

Mots-clés : économie, libéralisation, pauvreté 